INDO GLOBAL JOURNAL OF

PHARMACEUTICAL SCIENCES

ISSN 2249- 1023

\title{
Investigation of Cr (VI) Induced Oxidative Stress in Oryza sativa
}

\author{
Gauransh Jain, Aditi Khare, Sonali Dubey, Vibha Rani * \\ Department of Biotechnology, Jaypee Institute of Information Technology, A-10, Sector-62, Noida - 201307, UP, India
}

Address for Correspondance: Vibha Rani, vibha.rani@jiit.ac.in

Keywords Heavy

Metal Stress; $\mathrm{Cr}$

Toxicity; Reactive

Oxygen Species;

Anti-oxidant

Enzymatic Activity.

\begin{abstract}
The toxic effluents released untreated in the water bodies have led to the accumulation of toxic heavy metals (HM) like $\mathrm{Cr}$ in water bodies and soil. $\mathrm{Cr}$ accumulation is a cause of concern, especially in the submerged rice fields, which constitutes a staple diet of over $60 \%$ of Indian population. Cr metal toxicity in rice plants is related to the increased response of reactive oxygen species (ROS), which leads to oxidative stress and disorganization of lipids in cell membrane. On growing IR-64 Rice (Oryza sativa) in Hydroponics media (Hewitt media) under both control and $\mathrm{Cr}$ stressed conditions, results showed decreased growth in $\mathrm{Cr}$ exposed plants. HM stress induced severe changes in the secondary metabolite production of Oryza sativa, thus total phenolic and flavonoid content were found to be increased in $\mathrm{Cr}$ stressed plants. The total antioxidant activity was assessed based on the scavenging activity of the stable DPPH free radical, where the results showed significant difference in the inhibition activity. MDA, one of the lipid peroxidation products, was studied as a criterion for lipid oxidative stress. Cr exposure resulted in significant increase in its levels. An insight into the effects on the anti-oxidant enzymes were also analyzed by GPX and GST analysis, their overexpression suggested increased tolerance of plants in response to stress. This investigation will probably give an insight into the molecular mechanism involved in $\mathrm{Cr}$ (VI) Heavy metal stress reduction, which is to be explored further. (C) 2016 iGlobal Research and Publishing Foundation. All rights reserved.
\end{abstract}

Conference Proceedings: International Conference on Advances in Plant and Microbial Biotechnology (PMB2017); JIIT, Noida: February 02-04, 2017

Indo Global Journal of Pharmaceutical Sciences( ISSN 22491023 ; CODEN- IGJPAI; NLM ID: 101610675) indexed and abstracted in EMBASE(Elsevier), SCIRUS(Elsevier),CABI, CAB Abstracts, Chemical Abstract Services(CAS), American Chemical Society(ACS), Index Copernicus, EBSCO, DOAJ, Google Scholar and many more. For further details, visit http://iglobaljournal.com 